\title{
A Multicenter Cross- Sectional Survey on Physiotherapy Treatment Practices in Hemiplegic Shoulder Pain and Subluxation in Stroke Patients
}

\section{Muhammad Tahir Aslam* and Syed Shahzad Ali}

School of Physiotherapy, Dow University of Health Sciences Karachi, Pakistan.

*Corresponding Author: Muhammad Tahir Aslam, School of Physiotherapy, Dow University of Health Sciences Karachi, Pakistan.

Received: August 06, 2019; Published: August 20, 2019

\begin{abstract}
Objectives: To identify the current physiotherapy treatment practices in hemiplegic shoulder pain and subluxation in stroke patients. Material and Methods: A cross sectional survey was conducted on 151 Neuro musculoskeletal Physical Therapists, who fulfilled study inclusion criteria and working in different public and private Physiotherapy departments in Karachi, Pakistan by using Non probability purposive sampling Technique. The objective of the study was explained and informed consent was taken from all the Physical therapists taking part in this study. This study used a self-designed structured questionnaire that was pretested through a pilot survey on 25 subjects prior to assess their attitudes and treatment practices. The questionnaire had two sections, in the first section informations related to the participant's demographic, educational details and clinical experiences were asked. Whereas, in section two, informations related to their clinical practices were inquired. Statistical analysis was done through SPSS Version 21. The frequencies and percentages for all categorical variables were computed. Mean and standard deviation were calculated approximately for all continuous variables. P-value $<0.05$ will be considered statistically significant.

Results: The results of this survey show that majority of physical therapists having DPT as a graduation degree, in which females are significant higher than males from private clinical sectors working in musculoskeletal speciality, treat more than two patients of HSP and subluxation in a week. They are certified in Bobath and PNF techniques and use stabilization exercises, shoulder supports, electrical stimulation for treatment purposes. The subjects consider poor handling of patient as a limiting factor in therapy and suggest that HSP or subluxation affect quality of life of a patient.

Conclusion: It is suggested that majority of physical therapists in Karachi, Pakistan use shoulder supports, stabilization exercises, electrical stimulation, and are trained in PNF and Bobath therapies in management of HSP and subluxation in stroke survivors.

Keywords: Hemiplegic Shoulder Pain; Hemiplegia; Subluxation; CVA; Stroke
\end{abstract}

\section{Abbreviations}

CVA: Cerebrovascular Accident; HSP: Hemiplegic Shoulder Pain; PTs: Physical Therapists.

\section{Introduction}

Globally, hemiplegic shoulder pain (HSP) and sometimes subluxation are the most common and disabling impairments seen in post stroke survivors. The Frequency of hemiplegic shoulder pain ranges $70 \%$ or more in individuals suffering from stroke [1]. In United Kingdom, there is incidence of Hemiplegic Shoulder Pain of 200 per 100,000 population per year [2]. In Thailand, the occurrence of glenohumeral subluxation is reported to be $37 \%$ and is linked with hemorrhagic type of stroke, loss of proprioception sensation and Brunnstorm 'stage of arm recovery [3].
According to a systematic review in UK, there is no clear evidence that will report any connection between subluxation of glenohumeral joint and shoulder pain in stroke population [4].

The hemiplegic shoulder pain (HSP) delays rehabilitation and increases the financial expenses of management in people with stroke. Unfortunately, a very little is known about the incidence of hemiplegic shoulder pain in Pakistan [5].

But there is high possibility of presence of stroke in Pakistan due to increased chances of stroke in the society like high blood pressure, diabetes mellitus, smoking, more lipid concentration in blood than normal and lack of awareness with absence of management clinical practices [6]. As stated in an online survey conducted in UK, 
on the assessment, diagnosis and management of HSP, the investigators suggested that proper and careful assessment and diagnosis of cause of HSP, are essential for better outcomes in physiotherapy and occupational therapy management for HSP [7].

Hemiplegic shoulder pain (HSP) can affect as early as in first week after experiencing stroke. However, it is frequently observed within 8-10 weeks post stroke [8]. Similarly, It is reported that $30 \%$ of individuals suffer hemiplegic shoulder pain in the first year after cerebrovascular accident in Australia [9].

Main causes of HSP are spasticity in subscapularis and pectoralis major muscles, adhesive capsulitis of shoulder, alteration in sensitivity of affected upper extremity and subluxation of affected shoulder. The shoulder subluxation is often linked to the painful hemiplegic shoulder but it is not necessary that all patients after stroke and subluxation experience shoulder pain [10]. Moreover scapular dyskinesia, complex regional syndrome, central post stroke pain, and impingement syndromes can become other sources of HSP [11].

A national survey conducted in Netherland, which suggested that preventive measures, physiotherapy, mechanical approach and sometimes corticosteroids injections are important therapeutic efforts in managing the HSP [12].

In a systematic review on interventions treatment options for HSP, the authors from South Korea, point out that neuromuscular electrical stimulation is effective therapy for HSP when applied for three months [13]. Similarly, a study was conducted in USA for management of hemiplegic shoulder complex which revealed that neuromuscular electrical stimulation is effective in glenohumeral subluxation and taping/strapping may be beneficial in decreasing shoulder pain but it has never improved motor function or range of motion of affected upper limb [14].

A research was done in Pakistan about the role of supportive devices for treatment of HSP which suggested that proper positioning, family counseling, use of shoulder support and early usage of neuromusculoskeletal electrical stimulation were effective strategies for the prevention of subluxed shoulder. They also concluded that use of shoulder strapping within 24 hours is effective for delaying onset of HSP [15].

A review conducted in Pakistan to summarize the causes, assessment and management strategies of shoulder subluxation in stroke patients, in 2016. The authors advocated that the early usage of neuromuscular approaches, proper positioning, family's ed- ucation, kinesiology taping and robotic devices for cure of HSP. The robotic protocol was seemed to be effective for reduction in spasticity and prevent adhesive capsulitis of shoulder [16]. A randomized controlled study was performed in Taiwan to find the efficacy of application of kinesiology taping in shoulder pain in hemiplegia and functional results in sub-acute stroke patients. This study resulted that kinesiology taping might limit HSP and enhanced shoulder flexion but it had no role in improvement of upper extremity function [17].

It is apparent in a study in USA, that Bobath therapy, Brunnstorm and task specific for motor retraining, are beneficial in managing HSP but no one therapy proved to be more efficient than another [18]. In addition to these above mentioned techniques, proprioceptive neuromuscular facilitation approach for managing HSP is shown to be useful [19]. It is evident in a systematic review performed in Spain that physiotherapy treatment is necessary to stop or treat glenohumeral pain in post stroke patients and no specific physiotherapy technique is proved more better than others in HSP management [20].

\section{Study objectives}

To identify the current physiotherapy treatment practices used in hemiplegic shoulder pain and subluxation in stroke patients.

\section{Study rational}

Physical therapists play a major role in the management of stroke rehabilitation. To author's knowledge, in Pakistan, none of the study conducted earlier which discover physiotherapy treatment practices in hemiplegic shoulder pain and subluxation. This study explored current physiotherapy practices in the rehabilitation of hemiplegic shoulder pain and subluxation in Karachi, Pakistan.

\section{Literature Review}

According to a systematic review in UK on relationship stuck between the glenohumeral subluxation and HSP in stroke patients by Kumar P., et al. they found that there was no clear evidence that would report the connection between Hemiplegic Shoulder Pain and subluxation of glenohumeral joint in stroke population. They searched their required articles manually from different journals and also from MEDLINE, EMBASE, CINAHL and Cochrane library and found 148 researches. They chose 14 studies, out of which 7 had an association between HSP and shoulder subluxation and 7 did not find association. The writers had an opinion that this area lack high quality clinical research [4].

A hospital based cross sectional study was carried out on 100 individuals for determination of frequency and intensity of gle- 
nohumeral pain after stroke by Akhlaq U., et al. in Pakistan. They include males and females patients meeting the criteria of World Health Organization of stroke with shoulder pain and persons with chronic pain before stroke, rheumatic diseases or cognitive dysfunction were excluded. They came across that patients suffered from stroke, experienced glenohumeral joint moderate to severe pain in first year of stroke but it was not linked to primary cause of stroke and side of involvement.It was also found thatthe hemiplegic shoulder pain (HSP) delays rehabilitation and increases the financial expenses of management in people with stroke. Unfortunately, a very little is known about the incidence of hemiplegic shoulder pain in Pakistan [5].

An online survey conducted in UK, on the assessment, diagnosis and management of HSP by physiotherapists and occupational therapists, the investigators developed a questionnaire based on similar surveys of musculoskeletal and neurological practices, evaluated the literature and consulted with clinicians. They distributed this questionnaire online to physiotherapists and occupational therapists working in stroke rehabilitation settings. The authors included education, exercise and self management as therapeutic interventions. They concluded that proper and careful assessment and diagnosis of cause of HSP, are essential for better outcomes in physiotherapy and occupational therapy management for HSP [7].

A national survey conducted in Netherland in 2000 on management of shoulder pain in hemiplegia in which a postal questionnaire was sent to physical therapists, occupational therapists, neurologists, nursing home physicians and rehabilitation physicians from all rehabilitation centres with omission of pediatric and psychogeriatric centres. Each specific rehabilitation speciality had 100 individuals that formed a 500 subjects for survey. The examiners suggested that preventive measures, physiotherapy, mechanical approach and sometimes corticosteroids injections were important therapeutic efforts in managing the HSP [12].

In a systematic review in 2010 on interventions treatment options for HSP, the authors from South Korea, pointed out that neuromuscular electrical stimulation was successful therapy for HSP when applied for three months. Their investigation proved that shoulder pain was reduced in parallel of spasticity and subluxation. They used MEDLINE,EMBASE, CINAHL, PEDro and Cochrane to get eight RCT's including the criteria of stroke patients with shoulder pain, comparison of an intervention to another and shoulder pain as an outcome. The authors also stated in study that variation in shoulder pain was linked with alteration in lateral rotation of shoulder passively [13]. Similarly, a study was conducted in 2014, in USA for management of hemiplegic shoulder complex by Griffin Christine which revealed that neuromusculoskeletal or muscular electrical stimulation (NMES) was useful in subluxation and taping/strapping might be beneficial in reduction of shoulder pain but it had never improved motor function or range of motion of affected upper limb. In that study the researcher assessed the shoulder, trunk and pelvis of participants biomechanically before therapyand maintained passive shoulder flexion and abduction upto 90 degrees during therapy sessions. The shoulder subluxation was managed with neuromusculoskeletal electrical stimulation for six hours daily for six weeks with 5 days per week. It was also resulted in that research that trunk alignment, trunk control, positioning of scapula, pain prevention and NMES were treatment strategies for HSP [14].

A meta-analysis was done in Pakistan in 2012 by Khan M., et al. about the role of supportive devices for dealing with HSP in which investigators searched manually for the literature from PEDro, MEDLINE, CINAHL, PubMed, EMBASE, Scopus and Cochrane library. They included those articles which were RCT"s about hemiplegic individuals, human subjects related to this study. The instigators selected six articles among 87 in this study and suggested that proper positioning, family counseling, use of shoulder support and early usage of neuromuscular electrical stimulation were efficient strategies for the prevention of shoulder subluxation. They also concluded that use of shoulder strapping within 24 hours is effective for delaying onset of HSP [15].

A review conducted in Pakistan Razaq., et al. for summarize the reasons, assessment and management methods of shoulder subluxation in stroke patients, in 2016. The authors advocated the early usage of neuromuscular approaches, proper positioning, family's education, kinesiology taping and robotic devices for cure of HSP. The robotic protocol was seemed to be effective for reduction in spasticity and prevent adhesive capsulitis of shoulder [16].

In 2016, a randomized controlled study was performed in Taiwan on effects of kinesio-taping on shoulder pain in hemiplegia and functional results in sub-acute stroke patients. The aim of their study was to review the impact of kinesiology taping on HSP, functional outcomes of upper extremity and avoidance of soft tissue trauma in subacute stroke patient with HSP during therapy. The authors included forty four subacute stroke patients with hemiplegia and were separated randomely in two groups. The experimental group was treated with a 3 week Kinesiology Tape and conventional therapy for 5 days a week while the control group was managed with a 3 weel Sham Kinesio Tape with conventional rehabilitation for 5 days per week. This study resulted that kinesio taping might limit HSP and enhanced shoulder flexion but it had no role in improvement of upper extremity function, quality of life and daily functions [17]. 
Vasudevan and Brown made a study in USA in2014to see the effects of hemiplegic shoulder pain and focused on its diagnosis and management. They described different mechanical and neurological causes of HSP with its diagnosis through clinical and diagnostic imaging. They advised strapping, slings, TENS, FES, botulinum toxin injection, acupuncture, suprascapular nerve block and surgical and drug therapy for treatment of HSP. It was also apparent in a study that Bobath therapy, Brunnstorm and task specific for motor retraining, were beneficial in managing HSP but no one therapy proved to be more efficient than another [18].

Walsh K. did a study in UK in 2001 to find the evidence in dealing of pain in shoulder in subjects with stroke. In keeping with him, The ideal management of hemiplegic shoulder pain was to prevent it before happening in the first place. He also illustrated positioning, TENS, functional electrical stimulation, surgery, botulinum toxin injection, heat and cold therapy and drug therapy as the options for managing HSP. In addition to these above mentioned techniques, Bobath therapy, motor relearning program and proprioceptive neuromuscular facilitation approach for managing HSP were also shown to be useful [19].

A systematic review was performed in 2013 by Valero and Henarejos in Spain in which they wanted to find the the physiotherapy treatments that were most helpful in the avoidance and treatment of painful shoulder in hemiplegia. They searched about all available databases as well as a manual searching for clinical trials related to their objective. Only sixteen studies were selected and they made results. They advocated strapping the shoulder, slow-stroke back massage, profound dry needling, cold therapy, robotic system for therapy, TENS therapy, aromatherapy acupressure, FES and Bobath technique for HSP management. It was also evident that physiotherapy treatment was necessary to stop or treat shoulder pain in post stroke survivors but no specific physiotherapy technique was proved more better than others in HSP management [20].

I.Lindgren., et al. did a qualitative study in Sweden consisting of thirteen community-lodging persons (six women; median age 65 years) with constant pain in shoulder after stroke in 2017. The objective of study was to illustrate occurrence of shoulder pain after stroke, how it affected activities of daily living and identified consequences of intervention. They found that glenohumeral pain after stroke can direct to a variety of pain characteristics with radiation to the arm and hand and it often influenced one's personal care, activities of daily living negatively, which might guide to emotional responses. According to them, interventions by a multidisciplinary team might be needed for management the shoulder pain after stroke [21].
A literature review was accomplished by Clots. T., et al. to know the evidences about the physiotherapy methods used in treating shoulder pain in stroke patients in 2006. This study consisted of articles printed from 1997 to 2004 and alphabetically listed at the Medline, Lilacs, Pubmed and Cochrane databases. They chose patients diagnosed with acute or chronic stage and treated with physiotherapy interventions only. They excluded studies whose aim was to avoid and not managing the shoulder pain in stroke patients. The studies had not included which had not related to evaluate development of a shoulder pain after the head injury or argued only medical treatment and not physical therapy strategies. They selected 66 studies including systematic reviews, randomized controlled trials, case report or case series. The only twelve studies met the criteria. The canvassers suggested that electrical stimulation showed improved results in lessening the severity of shoulder pain and subluxation, as well as enhancing muscle function and joint range of motion of the upper extremities but it was not the best therapy. Other methods must be considered complementary treatment methods like positioning, handling, bandaging the shoulder and use of ultrasound, hot and cold therapies, feedback were regarded as supported therapies [22].

Vianna R., et al. presented a systemic review on the efficacy of therapeutic interventions in people with shoulder pain for more than six months duration after stroke. They looked for different databases for getting English articles from 1980 to 2012. The criteria of their study was participants were adults with a stroke at least six months back and they comprised of randomized controlled trials (RCT) that examined the usefulness of any therapeutic method for HSP. Ten studies were chosen from 226 articles following the criteria. The authors recommended that there was a therapy choice for shoulder pain for greater than 6 months after stroke to inject steroid injections into the joint or subacromial space. Electrical stimulation was considered successful as it would recover the level of subluxation and might useful in eliminating pain of shoulder in stroke subjects [23].

Mazen Al qahtani presented a narrative review in 2018 about current advancement in Physiotherapy interventions in Shoulder Subluxation in stroke patients. MEDLINE, EMBASE and Saudi Digital Library (SDL) were searched electronically from 1990 up to June 2017 to identify appropriate trials published in peer-reviewed journals in English language for that narrative review. The author concluded that shoulder slings, tapping techniques, arm troughs, lap boards and functional electrical stimulation were choice for management of HSP. There were other options like surgery, kinesiology tapping, but the evidence supporting these were not so decisive. Last but not least, ultrasound assessment had been found to 
exactly measure shoulder subluxation, and FES was the best choice in shoulder subluxation preventive as well as therapeutic [24].

In 2017, a systemic review conducted consisting of twenty two studies in India on rehabilitation methods for decreasing shoulder subluxation in post stroke hemiparesis, in which RCT's, Quasi experimental studies, having ischemic or hemorrhagic stroke in both males and females in any grade of shoulder subluxation were included. The main focus was on rehabilitation interventions such as orthosis, supports, slings, positional techniques, robotic therapy, exercise therapy and functional electric stimulation. The authors ruled out any case studies, case reports cohort or retrospective studies, traumatic subluxation or fractured shoulder or hemiparesis with head injury with therapeutic techniques like electroacupuncture. The intention of the study was to discover the evidences that disclosed the value of different rehabilitation interventions to overcome the subluxed shoulder in cerebrovascular accident. They found that no practice could reduce the shoulder subluxation in stroke patients efficiently and facilitate the upper limb recovery too. But, functional electrical stimulation proved itself better in reduction of shoulder subluxation in acute stages and shoulder supports or orthosis lessen it temporarily [25].

HC Hanger., et al. performed a single-blind prospective, randomized controlled stuy about shoulder strapping role in HSP in New Zealand in 2000. Their plan was to decrease or avoid shoulder pain and determination of range of motion and functional outcomes of affected shoulder. Ninety eight subjects were chosen at random in experimental (strapping) or control (without strapping) groups. The shoulder strapping was continued for whole time and was changed every 2-3 days for a total of six weeks or until discharge from hospital. It was resulted that strapping the shoulder did not produce effects in elimination of shoulder pain [26].

Nadler and Pauls completed a systematic review in UK in 2016 on finding out the role of shoulder orthoses in preventing or declining HSP and glenohumeral subluxation. They incorporated randomised randomized controlled trials and observational studies dealing with the consequences of application of a shoulder orthosis with adults suffering from stroke in English language. They rejected the studies in which researchers observed outcomes of application of a shoulder orthosis exclusively on balance and gait problems in stroke patients. Only eight studies were qualified on eligible criteria. Then the authors established a conclusion that orthoses reduced hemiplegic shoulder pain and tolerable with prolonged use but there was no evidence that subluxation and pain could be prohibited by instant application of orthoses after stroke [27].
A randomized controlled trial was completed in India by Hayner., et al. to assess the efficiency of California tri-pull taping (CTPT) method on HSP and subluxation after stroke in 2016. They picked up 30 individuals through randomization with acute stroke aged 35 to 70, with a minimum of $5 \mathrm{~mm}$ shoulder subluxation with pain in the affected upper limb [4], Mini Mental Status Examination (MMSE) score is higher than 23 and Brunstrom's stage 1 and 2 . They excluded subjects if they had MMSE score $<23$, any musculoskeletal disorder or any history of injury to the diseased upper extremity, dermal allergy from tape, Brunstorm's stage 3 and 4. Patients having a neurological disease except stroke and non cooperative patients were also taken out from study. They formed two equal groups of 15 individuals through randomization into a control group and an experiment group. All the participants got conventional neuro-rehabilitation which contained range of motion exercises, neuromuscular re-education, normalization of muscle tone, weight bearing training of the affected limb, functional and activities of daily living. The dyration of therapy was 45 minutes per day for 5 days a week for 6 weeks. In addition to that, the experimental group was treated with California tri-pull taping (CTPT) technique which used three portions of rigid tape, with a hard upward pull, to hold up the subluxed shoulder in stroke subjects. The researchers decided that California tri-pull taping method was used in decreaseing pain, improving active flexion range of shoulder and enhancing distal UE functional ability. It was also concluded that taping technique permits the patients to participate in all upper limb movements as well as all activities of daily living actively [28].

A Fil., et al. completed a prospective randomized controlled trial in 2010 on the competence of electrical stimulation in the avoidance of subluxed shoulder inferiorly and anteriorly in acute stroke patients in combination with Bobath techniques. Only the patients suffering from a stroke for the first time with a stroke history of maximum two days were enrolled in the study. The patients were excluded if they had another neurological disorder or a earlier disease of the shoulder or cardiac pacemaker. In that study, forty-eight stroke subjects were included who were eligible according to the inclusion criteria. The participants were indiscriminately divided into a study group and control group. Both groups were managed with acute stage physiotherapy exercises based on the Bobath concept. The study group was treated with high voltage pulsed galvanic stimulation of the supraspinatus and deltoid muscles. The authors reported that electrical stimulation combined with Bobath techniques was a successful method for prevention of subluxation of glenohumeral joint in stroke patients in acute stage [29].

A meta-analysis was done in China in 2018 to discuss the consequences of electrical stimulation (ES) in the management of 
shoulder pain in hemiplegia. The investigators searched for randomized controlled studies with a therapeutic period of at least two weeks and were related to comparison of electrical stimulation with sham stimulation or no stimulation for patients with HSP from eight databases. They excluded observational and retrospective studies and the studies involving subjects without a clear diagnosis of HSP and the studies without accessible data or contact with the authors. Only six studies met the criteria. The authors advocated that ES might be an effective pain reduction strategy for hemiplegic shoulder [30].

A study was fulfilled by Duangon s., et al. in Thailand in 2014 to note the role of interferential current stimulation (IFC) on shoulder pain and pain-free range of motion of the shoulder passively in people with hemiplegic shoulder pain. This was a placebo-controlled clinical trial and dual blinded by investigators. Thirty participants who were diagnosed with their first stroke were included to the study with more than 20 years old and pain in the diseased glenohumeral joint within 3 months of start of stroke. The patients also enrolled who had adequate communication abilities and normal light contact and pin-prick sensation on the affected shoulder; with Brunnstrom motor recovery stage of $1-3$, had no cognitive impairment and no history of arrhythmias, malignancy, cardiac pacemaker, no skin problems, wounds, or infections on the affected shoulder. The Patients were ruled-out if they could not complete the session. These 30 people were allotted two equal groups from first to last. The experimental group received Interferential current stimulation for 20 minutes with a frequency at $100 \mathrm{~Hz} 16$ in vector mode while the other faction obtained only placebo by disconnecting the lead wires of IFC equipment. It was confirmed that interferential current stimulation was beneficial for the pain relief during movement and enhanced the ROM of the shoulder passively in people with HSP in pain free range [31].

In 1994, a randomized controlled trial was performed by Faghri PD., et al. to estimate the efficiency of a functional electrical stimulation (FES) treatment to prevent glenohumeral joint subluxation and pain in stroke patients. They enrolled 26 stroke patients of both sexes in study through random allotment of two equal groups. They rejected patients with cardiac pace makers for their study. Both groups entertained with conventional physiotherapy for six weeks with additional functional electrical stimulation therapy to experimental group only. They concluded that the FES treatment was helpful in decreasing hemiplegic shoulder pain and subluxation and assisting in revival of arm function [32].

In Turkey, E. Koyuncu., et al. carried out a randomized controlled trial in 2009. The aim of the study was to know the evaluation of functional electrical stimulation (FES) for the therapy of subluxation and pain in shoulder in hemiplegic patients. The study contained a total of 50 appropriate patients with shoulder pain and subluxation due to stroke affecting on one side of the body. Patients having pacemaker in heart, deficit due to past contralateral stroke, a musculoskeletal shoulder pathology and uncooperative and epileptic patients were ruled out from the study. All subjects were randomly separated into two equal groups. The investigational group was applied FES treatment five times per day for one hour daily for 4 weeks to supraspinatus and posterior deltoid muscles on the hemiplegic side. They received a total of 20 sessions of therapy with addition of conventional therapy. The other group received only conventional rehabilitation management. The researchers advised that application of FES therapy with conventional treatment for managing shoulder subluxation and pain in hemiplegic patients is more useful than conventional treatment by itself [33].

A study was performed in 2017 by Lee., et al. to observe the efficiency of neuromuscular electrical stimulation (NMES) for the managing the shoulder subluxation after stroke. It was a meta analysis and systematic review in which Lee and colleagues searched different databases for all randomized controlled trials that assessed the effect of NMES and conventional therapy on subluxation of glenohumeral joint after cerebrovascular accident. The studies were also incorporated which used to overcome shoulder subluxation using surface electrodes and evaluated electrical stimulation of any type of stimulation in therapy. They ruled out studies in which the subjects with shoulder subluxation were associated with other neurological conditions like cerebral palsy, spinal cord injury. Eleven studies were included in that review after reviewed independently by two reviewers. The authors suggested that neuromuscular electrical stimulation when applied daily for one hour or more, diminished subluxation of shoulder for persons with acute/ subacute stoke. But it was not proved beneficial for individuals with chronic stroke for decreasing subluxation [34].

P. Pillatrini., et al. did a randomized controlled trial in 2019 to see effects of neuromuscular taping in hemiplegic shoulder pain with 32 stroke patients in Italy. They picked up patients with right or left hemiplegia due to ischemic or hemorrhagic stroke in addition to shoulder pain and also spasticity. They dropped patients from study if they had flaccidity, previous history of shoulder surgery or taking any NSAID or muscle relaxant drug. They divided participants into two groups. The investigational group treated with the application of Neuromuscular Taping and a physical therapy programme. The control group received only standard physical therapy programme. All subjects received therapeutic sessions for four weeks for forty five minutes in a day. They reported that the shoulder pain reduction and the improvement of range of motion were due to the application of an upper limb neuromuscular taping [35]. 
HeoM.Y. and colleagues finished a randomized controlled trial in 2015 in Republic of Korea to scrutinize the outcome on the shoulder joint of applying inelastic taping for acute stroke. The 36 subjects with stroke and shoulder pain and subluxation were recruited who had the same opinion to take part in the experiment. The experimental group had got inelastic taping with bed physical therapy. The control group received only bed physical therapy. The duration of therapy for both groups was eight weeks. The authors reported that the application of inelastic taping was helpful in reducing shoulder pain and subluxation for patients with acute stroke [36].

A study was carried out in 2015 in Kingdom of Saudi Arabia in which investigators wanted to know about the effects of Maitland's oscillatory mobilization technique in hemiplegic shoulder pain in acute stroke. They picked up 30 (10 females and 20 males) individuals with stroke involving on one side of the body. They formed two groups of 15 participants in each group as an experimental and a control group. In group A, 11men and 4 women received grade 1,2 of Maitland oscillatory technique with traditional physiotherapy treatment. The group B was comprised of 6 women and 9 men obtained only traditional physiotherapy treatment. Both these groups were treated three times in a week for 12 weeks. Then these researchers concluded that Maitland oscillatory grades 1,2 technique was proved helpful in decline of pain in rehabilitation of acute HSP [37].

In 2019, Praveen Kumar did a preliminary prospective cross sectional study to assess the effects of Lycra arm sleeves on the glenohumeral subluxation in people with chronic hemiplegia after stroke. The other objective of that research was also to see patients' awareness regarding the realism of applying the Lycra arm sleeve in these patients. He selected five patients aged greater than 18 years, with hemiplegic stroke resulting in one sided weakness. All subjects had a palpable gap in the lateral side of the shoulder (acromion greater tuberosity distance) and were able to sit upright. He removed participants with other neurologic conditions, traumatic brain injury, brain tumors, or other serious comorbidities from study. He educated the patients to wear the Lycra arm sleeve for seven hours per day for seven consecutive days. It was found that the decrease in mean acromion greater tuberosity distance would be due to wearing of Lycra arm sleeves which could be proved beneficial in management of shoulder subluxation in chronic stroke [38].

A systemic review and meta analysis was done by $\mathrm{H}$. Lee and S. M. Lim in Seoul, Republic of Korea in 2019 on evaluating evidence for the usefulness of acupuncture in lessening shoulder pain after stroke. They searched for many databases for controlled Trials till
March 2015. Three Korean medical databases were searched for Korean publications. They looked for the China National Knowledge Infrastructure database for Chinese studies. The authors found 188 relevant articles but only 12 studies from China met the eligibility criteria. The inclusion criteria for that study was only randomized, controlled trial (RCT) including patients diagnosed with shoulder pain after stroke. The studies were rejected if the study design did not report sufficient informations or not allow evaluation of acupuncture effects on shoulder pain after stroke. They also removed articles which had compared different types of acupuncture only. They found that both acupuncture and rehabilitation seemed more helpful than rehabilitation alone for management of hemiplegic shoulder pain in stroke but the results were uncertain [39].

Lee., et al. presented a systematic review in Seol, Republic of Korea to see the effects of acupuncture for shoulder pain after stroke in 2012. They looked for Randomized controlled trials (RCTs) describing efficacy of acupuncture for hemiplegic shoulder pain after stroke. All articles were printed between January 1990 and August 2009 and were obtained from the National Libraries of Medicine, different databases, Korean Medical database and Chinese database. They included the trials acupuncture therapy or compared acupuncture with other treatments and excluded trials that compared two different types of acupuncture. The seven studies met the criteria out of 453 and took in the study. The authors proposed that acupuncture was an important therapy and it could be considered as an adjuvant therapy for shoulder pain after stroke when used with exercise [40].

Pan., et al. executed a randomized controlled trial in China in 2017 for evaluation of modified wheelchair arm-support to lessen hemiplegic shoulder pain and diminish pain frequency in stroke patients. They incorporated 120 individuals of ischemic or hemorrhagic stroke with shoulder pain aged between 20 to 85years.All subjects had healthy mental status, and Brunnstrome scale I or II. They took away 35 individuals from the study due to not following their exclusion criteria due to presence of brain injury, thalamic lesions or peripheral neuropathy. Participants were removed from study who had a history of cervical spondylosis, fracture or injury or adhesive capsulitis at the shoulder without stroke. The authors came to know after compilation of results that use of the modified wheelchair arm-support could decrease hemiplegic shoulder pain and it also decreased the occurrence of pain in stroke patients. It was also proved that it might also enhance muscular function of upper limb, daily activities' performance and quality of life when compared to using an ordinary wheelchair [41].

Kim., et al. performed a randomized controlled trial in Republic of Korea to evaluate the consequences of a sling exercise on 
shoulder subluxation in stroke patients in 2012. The sling exercise therapy used active-exercise treatment with a sling to decrease neuromuscular disorders. Thirty-four patients who had stroke for over nine months, and were generally recovered, were the subjects of this study. All individuals had shoulder subluxation without history of diabetes or other diseases which could damage peripheral nerves. All participants had no fracture, trauma on the affected side. The participants were randomly assigned two same groups which experienced traditional physical therapy exercises once per day for half an hour, five times a week for eight weeks. The experimental group was treated with sling exercise therapy for 30 minutes a day in addition to physiotherapy exercises. The authors suggested that the sling exercise was beneficial in decreasing the severity of hemiplegic shoulder subluxation [42].

Dohle., et al. accomplished a pilot study in which usefulness of robotic protocol for therapy of subluxation of shoulder in patients with chronic stroke was assessed in 2013. The study comprised of eighteen patients with chronic stroke in which 8 were women and 10 men. They were enrolled in the study on inclusion criteria having shoulder instability from more than 12 months in the chronic phase after stroke. The investigators removed subjects with intact shoulder joint, who were unable to follow commands and had a fixed contracture in any joint of the affected upper extremity. All subjects were managed with robotic training for six weeks, with 3 times in a week on alternative days using the linear shoulder robot. During the therapy, the patient was seated in a relaxed position, and compensatory body movements were minimized by using a seatbelt. Throughout the session, the patient was supervised by a skilled therapist. The patient was ordered to move between targets by following visual feed-back provided on a computer screen by a yellow ball. The subjects performed three sets of 320 repetitions of vertical arm movements and horizontal arm movements during each session. Both the antigravity and gravity eliminated movement training procedures were alternated across sessions. It was resulted in that study that the robotic protocol was a better treatment for shoulder subluxation in chronic stroke patients [43].

A systematic review was performed by Kumar., et al. to find risk factors for shoulder subluxation in inferior direction following stroke in 2010. They looked for different databases and the Cochrane Library to get relevant literature. They included any study related to patients with stroke and subluxation with particular patient population, sufficient sample size and clear eligibility criteria for subjects and sample selection method. Only eight studies met their criteria after independent critical appraisal. The investiga- tors concluded that shoulder subluxation after stroke could be prevented by strengthening rotator cuff muscles and improving muscular control around the shoulder complex in functional practice sessions [44].

A pilot, randomized controlled trial was in 2014 done by Wilson RD and colleagues on setting up the efficacy of peripheral nerve stimulation (PNS) therapy for pain elimination in patients with chronic shoulder pain in hemiplegia. They included 21 yrs or older adults with stroke and shoulder pain on the affected side with duration of HSP was more than 3 months. The exclusion criteria consisted of history of recurrent skin infections or insensate skin or taking one or more opioids or nonopioid analgesics. Those patients who were daily taking of pain medications for any other chronic pain or taking intra-articular or subacromial steroid injections to the shoulder in the previous 3 months or depressed or impaired cognition also excluded from study. They incorporated twenty five patients into their study and allotted them two groups through randomization. The experimental group consisted of 13 patients and were treated with peripheral nerve stimulation therapy with a single lead for 3 weeks while the second group received only usual care of physiotherapy treatment only for 3 weeks. They concluded that Short-term PNS was a safe and sound and successfully reduced the shoulder pain [45].

Shin and Lee performed a pilot randomized controlled trial to determine the benefits of Aromatherapy Acupressure on Hemiplegic Shoulder Pain and Motor Power in Stroke Patient in 2007. The rationale of that study was to find if aromatherapy acupressure, was helpful in reduction of hemiplegic shoulder pain and enhancing muscular power in stroke patients. Thirty stroke patients with hemiplegic shoulder pain were enrolled in the study who qualified inclusion criteria. The inclusion criteria was HSP with stroke with equal or below Grade 3 in muscle power of hemiplegic upper extremity without signs of allergy from aromatherapy, and agreed to the use of aromatherapy. They removed participants from study who had shoulder pain without stroke like shoulder pain due to vertebral origin, visceral origin, fracture or infected arthris. The investigators randomly allotted two different but equal groups to participants. The group one was treated with aromatherapy acupressure for 20-minute session, two times a day for 2 weeks, giving a total of 28 treatments. The subjects in second group were treated only with Acupressure-only treatment (dry acupressure). It was resulted from the study that aromatherapy acupressure decreased HSP in stroke patients and did so more efficiently than acupressure alone [46]. 


\section{Operational definitions}

- Hemiplegia: It is defined as complete or incomplete paralysis of one side of face, arm, leg unaided by sensory problems, dysphasia and visual field defects [47].

- Hemiplegic shoulder pain: It is a frequent problem after stroke and is described as sharp, stabbing pain which is more common on movement than at rest [48].

- Stroke: Stroke was defined as "rapidly developing clinical signs of focal(or global) disturbance of cerebral function lasting more than 24hours (unless interrupted by surgery or death) with no apparent cause other than of vascular origin" [49].

- Shoulder subluxation: It is defined as increased translation of head of humerus with regard to the glenoid fossa [50].

\section{Material and Methods}

- Study design: Cross sectional survey

- Sampling Technique: Non probability, purposive sampling Technique

- Sample Size: Sample size of 151was calculated using OPEN EPI version 3.0 with a hypothesized frequency of 83\%(Physiotherapist's responses on Physiotherapy management on HSP and subluxation) confidence limits of $6 \%$, design effect of $1 \%$ and confidence level of $95 \%$.(12)

- Study Duration: Three Months after the approval of synopsis.

Sample description

Inclusion criteria

- Both Male and female Physical therapists

- Working in Neurological \& Musculoskeletal Physiotherapy IPD or OPD

- $\quad$ Physical therapists who are involved in the management of patient with hemiplegic shoulder pain and subluxation

\section{Exclusion criteria}

- Undergraduate physical therapists

- Physical therapy Technicians

- Physical therapist who are not involve in patients care

- Clinical Physical therapists who do not treat patients with stroke.

\section{Study settings}

"Twelve Physiotherapy clinical settings from Public and Private hospitals/clinics in Karachi are selected for this study."
Questionnaire design

This study used a self-designed structured questionnaire that was pre-tested through a pilot survey on 25 subjects and updated accordingly. The questionnaire had two sections, in the first section information related to the participant's demographic, educational details and clinical experiences were asked. Whereas, in section two, information related to their clinical practices was inquired.

\section{Data collection}

Data was collected from participants who fulfilled our inclusion and exclusion criteria. A structured questionnaire was given and the purpose of the study explained and informed consent had been taken from all the physical therapist taking part in that study.

\section{Ethical considerations}

The study was conducted after the approval of synopsis from the institutional review board of Dow University of Health Sciences (IRB-1178/DUHS/APPROVAL/2018).

\section{Data analysis}

Statistical analysis was done through SPSS Version 21. The frequencies and percentages for all categorical variables were computed. Mean and standard deviation were calculated approximately for all continuous variables.

\section{Results}

The results of table 1 showed the demographic details of total physiotherapists participating in study were 151 with mean age 30 years. Females(53\%) were greater in number than males(46) with Doctor of Physical Therapy qualification(64.2\%) followed by Masters in physiotherapy(27.2\%). Most of the partaker were specialist in musculoskeletal physiotherapy(50\%) followed by neurological physiotherapy(45.0\%) with area of clinical practice was from private sector(62.3). Greater part of subjects had more than three years of clinical experience(52.3\%).

The study conclusions suggested that majority of the physiotherapists included in study treated 2 patients (59\%) in last four weeks and the type of stroke they had seen was ischemic stroke patients (51.7\%) next to hemorrhagic (47\%). Numerous subjects had managed patients with hemiplegic shoulder pain frequently (51\%) and afterward rarely(39.1\%) and they had attended workshops regarding HSP therapy(65.6\%). A lot of participants received certification or training in proprioceptive neuromuscular facilitation technique (55\%) followed by Bobath therapy (44.4\%) for management of HSP and subluxation of shoulder (Table 2). 


\begin{tabular}{|c|c|c|}
\hline Variables & & N (\%) \\
\hline \multicolumn{3}{|l|}{ Age } \\
\hline \multicolumn{3}{|c|}{ Mean \pm sd: $30.19 \pm 6.53$} \\
\hline \multicolumn{3}{|l|}{ Gender } \\
\hline & Male & $70(46.4)$ \\
\hline & Female & $81(53.6)$ \\
\hline \multicolumn{3}{|c|}{ Qualification } \\
\hline & BSPT & $13(8.6)$ \\
\hline & DPT & $97(64.2)$ \\
\hline & Masters & $41(27.2)$ \\
\hline \multicolumn{3}{|l|}{ Speciality } \\
\hline & Neurology & $68(45.0)$ \\
\hline & Musculoskeletal & $76(50.3)$ \\
\hline & Others & $7(4.6)$ \\
\hline \multicolumn{3}{|c|}{ Area of Clinical Practice } \\
\hline & Public & $57(37.7)$ \\
\hline & Private & $94(62.3)$ \\
\hline \multicolumn{3}{|c|}{ Years of Experience (years) } \\
\hline & $0 . .6$ & $1(0.7)$ \\
\hline & $<1$ & $14(9.3)$ \\
\hline & $1-2$ & $37(24.5)$ \\
\hline & $2-3$ & $20(13.2)$ \\
\hline & $>3$ & $79(52.3)$ \\
\hline
\end{tabular}

Table 1: Characteristics of study Population.

The outcome in Table 3 indicated that majority of the individuals taking part in study assessed hemiplegic shoulder pain and subluxation through $\mathrm{x}$ rays $(60.3 \%)$ from private sector $(64.8 \%)$ after that sulcus sign(59.6\%) from private sector(62.2\%). Several physiotherapists thought that common causes of hemiplegic shoulder pain and subluxation were poor handling (58.3\%) from private sector (72.7\%) afterward muscle tone(49.05\%) from private sector(56.8\%). Mostly participants used stabilization exercises (66.9\%) from private sector (58.45) subsequently by shoulder support(62.9\%) from private sector(64.25) followed by neuromuscular electrical stimulation (41.15) from private sector (69.45) as treatment options for management of hemiplegic shoulder pain and subluxation. Many of persons incorporated in study marked poor handling of patients(60.9\%) from private sector $(62.0 \%)$ followed by poor patient compliance $(41.1 \%)$ from private sector(53.2\%) as limiting factors in treatment of hemiplegic shoulder pain and subluxation. Most of the participants described that quality of life(82.8\%) of the patients from private sector was

\begin{tabular}{|c|c|c|c|c|}
\hline \multirow{3}{*}{ Variables } & & \multicolumn{3}{|c|}{ Clinical setting } \\
\hline & & & Public & Private \\
\hline & & N (\%) & N (\%) & N (\%) \\
\hline \multirow{4}{*}{$\begin{array}{l}\text { Stroke } \\
\text { patients } \\
\text { treated in Last } \\
4 \text { weeks }\end{array}$} & 2 & $90(59.6)$ & $34(37.8)$ & $56(62.2)$ \\
\hline & 3 & $36(23.8)$ & $11(30.6)$ & $25(69.4)$ \\
\hline & 4 & $10(6.6)$ & $4(40.0)$ & $6(60.0)$ \\
\hline & 5 & $15(9.9)$ & $8(53.3)$ & $7(46.7)$ \\
\hline \multirow{3}{*}{$\begin{array}{l}\text { Type of Stroke } \\
\text { patients }\end{array}$} & Hemorrhagic & $71(47.0)$ & $27(38.0)$ & $44(62.0)$ \\
\hline & Ischemic & $78(51.7)$ & $29(37.2)$ & $49(62.8)$ \\
\hline & Others & $2(1.3)$ & $1(50.0)$ & $1(50.0)$ \\
\hline \multirow{4}{*}{$\begin{array}{l}\text { Frequency } \\
\text { of Treating } \\
\text { Hemiplegic } \\
\text { shoulder pain }\end{array}$} & Never & $4(2.6)$ & $1(25.0)$ & $3(75.0)$ \\
\hline & Rarely & $59(39.1)$ & $23(39.0)$ & $36(61.0)$ \\
\hline & Frequently & $77(51.0)$ & $26(33.8)$ & $51(66.2)$ \\
\hline & Always & $11(7.3)$ & $7(63.6)$ & $4(36.4)$ \\
\hline \multirow{2}{*}{$\begin{array}{l}\text { Workshop } \\
\text { attended } \\
\text { regarding the } \\
\text { management } \\
\text { of HSP }\end{array}$} & No & $52(34.4)$ & $20(38.5)$ & $32(61.5)$ \\
\hline & Yes & $99(65.6)$ & $37(37.4)$ & $62(62.6)$ \\
\hline \multirow{5}{*}{$\begin{array}{l}\text { Certificate/ } \\
\text { Training } \\
\text { received }\end{array}$} & Bo bath & $67(44.4)$ & $26(38.8)$ & $41(61.2)$ \\
\hline & $\begin{array}{c}\text { Motor } \\
\text { relearning } \\
\text { program }\end{array}$ & $56(37.1)$ & $27(48.2)$ & $29(51.8)$ \\
\hline & $\begin{array}{c}\text { Kinesiology } \\
\text { Taping }\end{array}$ & $42(27.8)$ & $14(33.3)$ & $28(66.7)$ \\
\hline & $\begin{array}{l}\text { Propriocep- } \\
\text { tive Neuro- } \\
\text { muscular } \\
\text { facilitation }\end{array}$ & $83(55.0)$ & $32(38.6)$ & $51(61.4)$ \\
\hline & $\begin{array}{c}\text { Dry Needling } \\
\text { Techniques }\end{array}$ & $11(7.3)$ & $3(27.3)$ & $8(72.7)$ \\
\hline
\end{tabular}

Table 2: Description of Participants Clinical Expertise.

affected by hemiplegic shoulder pain and subluxation and again these all worked in private setting(62.4\%).

\section{Discussion}

The aim of this survey is to explore the physiotherapy treatment practices used in the management of hemiplegic shoulder pain and subluxation in stroke patients in the Karachi, Pakistan. The response rates proposed that physiotherapists consider hemiplegic shoulder pain as an essential issue. The questionnaire was sent to musculoskeletal and neurological physiotherapists in public and 


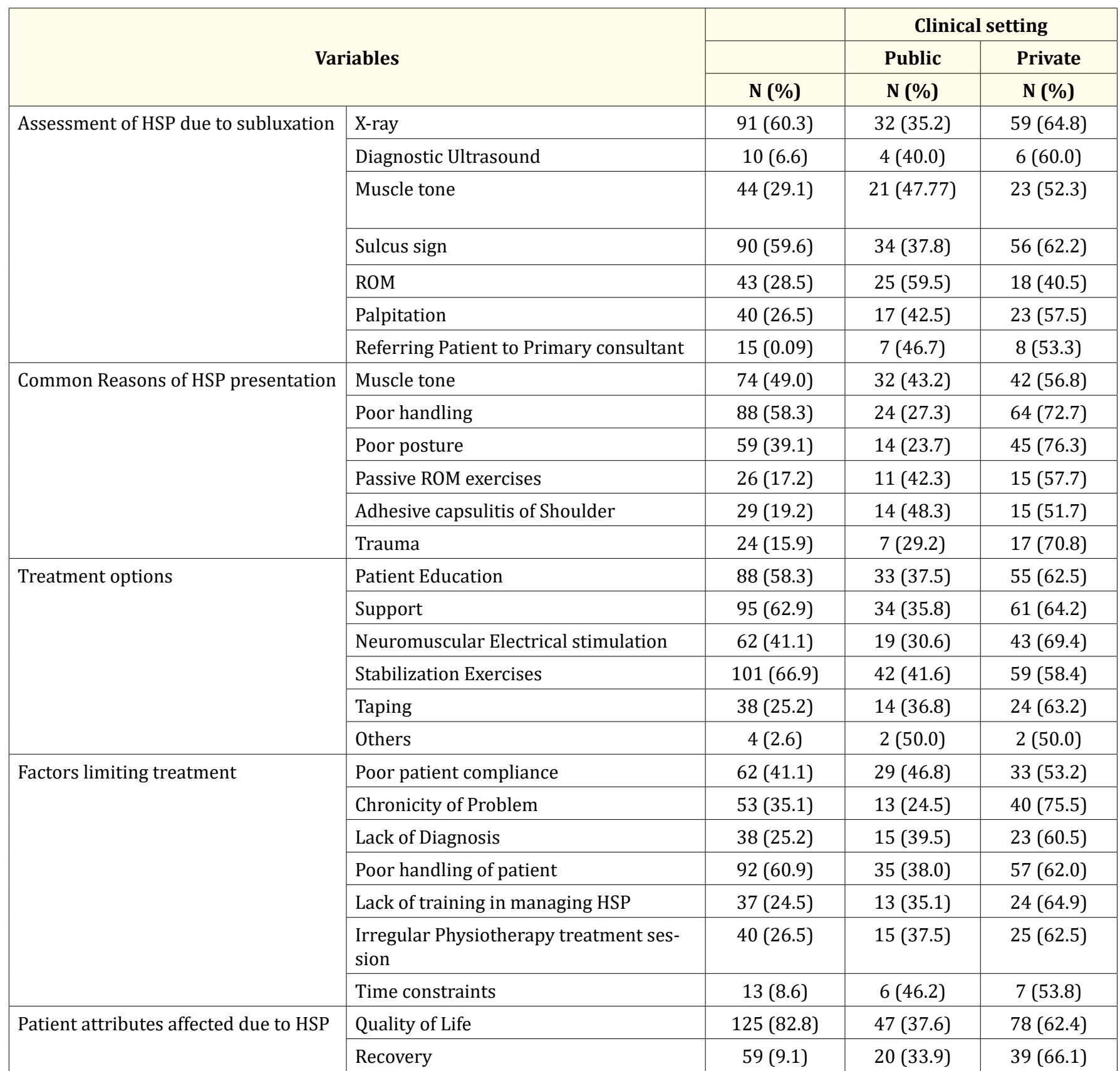

Table 3: Percentage distribution of Patients' attitudes towards assessment.

private hospitals and rehabilitation clinics because it was assumed that these physiotherapists regularly manage this problem. It was not surprising that the survey reported the application of many different therapies or combination of therapies for management of HSP and subluxation.

It is evident in this study that physiotherapists taking part in study who work in different private clinics or hospitals are much more than physiotherapists working in public sector clinical settings. It may be due to shortage of neuromusculoskeletal physiotherapists in Government sector in Karachi. The number of female participants is relatively larger than males with significant more than 3 three years clinical experience.
It is showed that most of the participants treated two patients ofshoulder pain and subluxation in stroke patients in last four weeks and they have experienced patients with ischemic type of stroke. Majority of them frequently manage hemiplegic shoulder pain and subluxation in stroke patients but others rarely treat this kind of patients which may be due to information bias from participants or due to their less experience. The data demonstrates that most of participants have got certification or training in proprioceptive neuromuscular facilitation technique and Bobath therapy for dealing with hemiplegic shoulder pain and subluxation. The two studies done by Vasudevan J, Browne B. and Walsh K proved that bobath therapy and proprioceptive neuromuscular facilitation 
technique play an effective role in managing HSP and subluxation respectively $[8,19]$.

The significant high number of physiotherapists who have participated in study, diagnose hemiplegic shoulder pain and subluxation through $x$ ray and sulcus sign. A study done by Stolzenberg D and Siu G. demonstrated that diagnose of HSP and subluxation was made clinically but sulcus sign and $\mathrm{x}$ ray(less important) played a role in diagnosis [51]. A major portion of subjects in this study give opinion that poor handling and muscle tone are the reasons for HSP and subluxation. L Turner-Stokes and D Jackson illustrated in a study that flaccidity and spasticity both cause HSP in stroke patients The authors also state that trauma to the shoulder joint can be prevented by proper positioning and handling in hemiplegic shoulder pain and subluxation cases [52]. Numerous subjects reported that stabilization exercises, shoulder support and neuromuscular electrical stimulation are (in that order) best therapeutic choices for hemiplegic shoulder pain and subluxation. Brook MM. and fellows advised in their study that different shoulder supports provideusefulness and specificity of shoulder support to decrease subluxation after hemipiegia [54]. Several studies like Qui H., et al., and Faghri PD et al. advised application of electrical stimulation (NMES/FES) for prevention or management of HSP or shoulder subluxation $[30,32]$.

The subjects consider that poor handling of patient by caregiver and poor patient compliance are the main limiting factors in rehabilitation of HSP and subluxation in stroke patients. There is a concensus of most of participants that quality of life of patient is disturbed by hemiplegic shoulder pain and subluxation. AdeyWakeling., et al. advocated in their study that Hemiplegic shoulder pain decreases quality of life of patients at 12 months [54].

Results of present study are compared with the two studies performed in UK [7] and Netherland [12]. In an online survey done in UK on the management of hemiplegic shoulder pain and sub- luxation, patient's education, self-management and exercises are therapeutical interventions. In Netherland, results of a national survey indicates that preventive measures and physiotherapy interventions were found effective in management of HSP. Both of these survey support the findings of present study.

\section{Strengths and weakness}

The strength of this study is that it is a first effort on physiotherapy treatment practices in hemiplegic shoulder pain and subluxation in Karachi, Pakistan. According to author's knowledge, no study has been done on this specific topic in Pakistan previously. Moreover, this study has been conducted from physiotherapists working in both private and public clinical settings and hospitals of Karachi. Additionally, there is a great deficiency of literature regarding cross sectional observational studies on physiotherapy treatment practices in hemiplegic shoulder pain and subluxation in stroke patients worldwide and in Pakistan too. So, this study is an effort to pay attention of other researchers to work on this important clinical issue in Pakistan.

It must be recognized that the subject's verbal reports about clinical practice may be different from their actual practice. Therefore, further studies are required using other methods to verify how these theoretical beliefs are applicable in clinics practically. Furthermore, this study only included Physiotherapists from public or private sectors from Karachi and lacks a large number of participants from all over the Pakistan. Therefore it does not cover whole physiotherapy population of Pakistan on physiotherapy treatment in HSP and shoulder subluxation in stroke patients. Future studies will include a large sample size from all over Pakistan to give a strong opinion of physiotherapists on this topic.

Conclusion

It is suggested that majority of physical therapists in Karachi, Pakistan use shoulder supports, stabilization exercises, electrical stimulation and are trained in PNF and Bobath therapies in management of HSP and subluxation in stroke survivors.

\section{HSP SURVEY QUESTIONNAIRE}

\section{STUDY TITLE: A MULTICENTRE CROSS SECTIONAL SURVEY ON PHYSIOTHERAPY TREATMENT PRACTICES IN HEMIPLEGIC SHOULDER PAIN AND SUBLUXATION IN STROKE PATIENTS.}

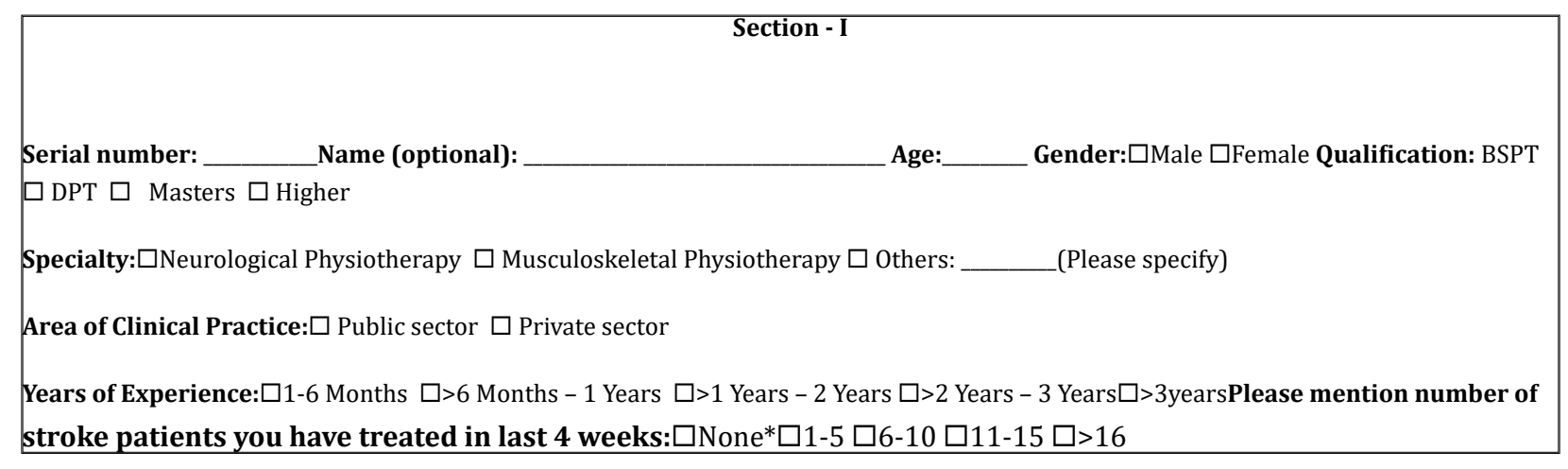


Section - II

1. Type of stroke patient you commonly treat:

$\square$ Hemorrhagic $\square$ Ischemic $\square$ Others:

2. How frequently you encounter hemiplegic shoulder pain (HSP):

$\square \quad \square$ Never $\square$ Rarely $\square$ Frequently $\square$ Always

3. Did you receive any update regarding to HSP management in past one year?

$\square \quad \square$ Yes $\square$ No

4. If you mark Yes, against the question \# 3 then please specify:

$\square \quad \square$ In-service training $\square$ clinical supervision $\square$ Reading related literature $\square$ others:

5. Please mention if you get any certification or training in the following neurological physiotherapy techniques for the management of stroke / HSP:

$\square \quad$ (You can mark more than one option)

$\square \quad \square$ Bobath $\square$ Motor Relearning Program $\square$ Kinesiology Taping

$\square \quad \square \quad$ Proprioceptive Neuromuscular Facilitation Techniques $\square$ Dry Needling Techniques $\square$ Others: Specify)

6. How do you assess patient with Hemiplegic shoulder pain due to subluxation:

$\square \quad$ (You can mark more than one option)

$\square \quad \square$ X-ray $\square$ Diagnostic Ultrasound $\square \quad$ Muscle Tone $\square \quad$ Sulcus Sign $\square$ ROM $\square$ Palpation

$\square \quad$ By referring patient to primary consultant

7. On average how long, it usually required to treat HSP?

$\square \quad$ 1-2 weeks $\square>2$ weeks - 3 weeks $\square>3$ weeks - 4weeks $\square>$ 4weeks - 5weeks $\square>5$ weeks

8. What treatment option usually considered in HSP?(You can mark more than one option)

$\square \square$ Patient Education $\square$ Slings/ Support $\square$ Neuromuscular Electrical Stimulation $\square$ stabilization exercises $\square$ Taping $\square$ others (Please specify

9. What factors limit in the treatment of HSP?

(You can mark more than one option)

$\square \quad \square$ Poor patient compliance $\square$ Chronicity of problem $\square$ Lack of diagnosis

Poor handling of patient during transfer $\square$ Lack of training in managing HSP $\square$ Irregular physiotherapy treatment session $\square$ Time constraint

10. Please mention that which of the following patient attribute affected due to HSP (you can mark more than one options)

$\square \quad$ Quality of Life $\square$ Recovery $\square \square$ others:

11. Which of the following are the common reasons of HSP patients present with: (you can mark more than one options)

$\square \quad \square$ Muscle Tone $\square$ Poor Handling $\square$ Poor Posture $\square$ Passive Range of Motion Exercises

$\square \quad$ Adhesive capsulitis $\square$ Trauma $\square$ Others:

12. Please specify if HSP results in any degree of impact on patient functional recovery:

$\square \quad \square$ Not Any $\square$ Mild $\square$ Moderate $\square$ Severe

Citation: Muhammad Tahir Aslam and Syed Shahzad Ali. "A Multicenter Cross- Sectional Survey on Physiotherapy Treatment Practices in Hemiplegic

Shoulder Pain and Subluxation in Stroke Patients". Acta Scientific Orthopaedics 2.9 (2019): 28-42. 


\section{Bibliography}

1. McKenna L. "Hemiplegic shoulder pain: defining the problem and its management". Disability and Rehabilitation 23.16 (2001): 698-705.

2. Davidson I and Waters K. "Physiotherapists Working with Stroke Patients". Physiotherapy 86.2 (2000): 69-80.

3. Seuthanapornkul S., et al. "Post stroke shoulder subluxation and shoulder pain: A cohort multicentrestudy". Journal of the Medical Association of Thailand 91.12 (2008): 1885-1893.

4. Kuar P., et al. "Association between glenohumeral subluxation and hemiplegic shoulder pain in patients with stroke". Physical Therapy Reviews 18.2 (2013): 90-100.

5. Khan A., et al. "Frequency and intensity of shoulder pain after stroke:a hospital based study". Pakistan Armed Forces Medical Journal 66.1 (2016): 71-74.

6. Khealani B and Wasay M. "The Burden of Stroke in Pakistan". International Journal of Stroke 3.4 (2008): 293-296.

7. Kumar P., et al. "Assessment, diagnosis and management of hemiplegic shoulder pain: A UK-Wide online survey of physiotherapy and occupational therapy practice". In: British Pain Society, Harrogate, UK (2016): 10-12.

8. Roy C., et al. "Shoulder pain in acutely admitted hemiplegics". Clinical Rehabilitation 8.4 (1994): 334-340.

9. Adey-Wakeling Z., et al. "Incidence and Associations of Hemiplegic Shoulder Pain Post stroke: Prospective Population-Based Study". Archives of Physical Medicine and Rehabilitation 96.2 (2015): 241-247.

10. Murie-Fernández M., et al. "Painful hemiplegic shoulder in stroke patients: Causes and management". Neurología (English Edition) 27.4 (2012): 234-244.

11. Wilson R and Chae J. "Hemiplegic Shoulder Pain". Physical Medicine and Rehabilitation Clinics of North America 26.4 (2015): 641-655.

12. Snels I., et al. "Treatment of hemiplegic shoulder pain in the Netherlands: results of a national survey". Clinical Rehabilitation 14.1 (2000): 20-27.

13. Koog Y., et al. "Interventions for hemiplegic shoulder pain: Systematic review of randomised controlled trials". Disability and Rehabilitation 32.4(2010): 282-291.

14. Griffin C. "Management of the Hemiplegic Shoulder Complex". Topics in Stroke Rehabilitation 21.4 (2014): 316-318.

15. Khan Muhammad., et al. "Reviewing The Role Of Supportive Devices For The Treatment Of Hemiplegic Shoulder Pain". Medical Channel 19.3 (2012): 8-13.
16. Razaq Sarah and AzamRathore Farooq. "An overview of pathophysiology, assessment and management strategies of post stroke shoulder subluxation,.". Pakistan Journal of Neurological Sciences 11.3 (2016).

17. Huangyc YC., et al. "Effect of kinesiology taping on hemiplegic shoulder pain and functional outcomes in subacute stroke patients: a randomized controlled study". European Journal of Physical and Rehabilitation Medicine 52.6 (2016): 774-781.

18. Vasudevan J and Browne B. "Hemiplegic Shoulder Pain". Physical Medicine and Rehabilitation Clinics of North America 25.2 (2014): 411-437.

19. Walsh K. "Management of shoulder pain in patients with stroke". Postgraduate Medical Journal 77.912 (2001): 645649.

20. Ramos-Valero L and Meseguer-Henarejos. "A physiotherapy treatment for patients with painful shoulder after stroke: a systematic review". Fisioterapia 35.5 (2013):214-223.

21. Lindgren I., et al. "Shoulder pain after stroke - experiences, consequences in daily life and effects of interventions: a qualitative study". Disability and Rehabilitation 40.10 (2017): 1176-1182.

22. Klotz T., et al. "Physiotherapy treatment in hemiplegic shoulder pain in stroke patients-Literature Review”. Acta Fisiátrica 13.1 (2006): 12-16.

23. Viana R., et al. "Evidence for Therapeutic Interventions for Hemiplegic Shoulder Pain During the Chronic Stage of Stroke: A Review". Topics in Stroke Rehabilitation 19.6 (2012): 514522.

24. Alqahtani M. "Physical Therapy intervention in post stroke shoulder subluxation: A narrative review, Majmaah".Journal of Health Sciences 6.1 (2018).

25. Kamal Narayan Arya., et al. "Rehabilitation methods for reducing shoulder subluxation in post-stroke hemiparesis: a systematic review".Topics in Stroke Rehabilitation (2017).

26. Hanger H., et al. "A randomized controlled trial of strapping to prevent post-stroke shoulder pain". Clinical Rehabilitation 14.4 (2000): 370-380.

27. Nadler M and Pauls M. "Shoulder orthoses for the prevention and reduction of hemiplegic shoulder pain and subluxation: systematic review". Clinical Rehabilitation 31.4 (2016): 444453.

28. Hayner K., et al. "The California tri-pull taping method in the treatment of shoulder subluxation after stroke: A randomized clinical trial". North American Journal of Medical Sciences 8.4 (2016): 175-182. 
29. Fil A., et al. "The effect of electrical stimulation in combination with Bobath techniques in the prevention of shoulder subluxation in acute stroke patients". Clinical Rehabilitation 25.1 (2010): 51-59.

30. Qiu H., et al. "Electrical stimulation in the treatment of hemiplegic shoulder pain". American Journal of Physical Medicine and Rehabilitation 99.9 (2018): 1730-1739.

31. Duangporn S-a., et al. "Effect of Interferential Current Stimulation in Management of Hemiplegic Shoulder Pain,". Archives Of Physical Medicine And Rehabilitation 95.8 (2014): 1441-1446.

32. Faghri PD., et al. "The effects of functional electrical stimulation on shoulder subluxation, arm function recovery, and shoulder pain in hemiplegic stroke patients". Archives of Physical Medicine and Rehabilitation 75.1 (1994): 73-79.

33. Koyuncu E., et al. "The effectiveness of functional electrical stimulation for the treatment of shoulder subluxation and shoulder pain in hemiplegic patients: A randomized controlled trial". Disability and Rehabilitation 32.7 (2009): 560566.

34. Lee J., et al. "Effectiveness of neuromuscular electrical stimulation for management of shoulder subluxation post-stroke: a systematic review with meta-analysis". Clinical Rehabilitation 31.11 (2017): 1431-1444.

35. Pillastrini P., et al. "Effectiveness of neuromuscular taping on painful hemiplegic shoulder: a randomised". Clinical Trial (2019).

36. Heo M., et al. "Influence of the application of inelastic taping on shoulder subluxation and pain changes in acute stroke patients". Journal of Physical Therapy Science 27.11 (2015): 3393-3395.

37. AlAmoudi KO., et al. "Effect of Maitland's oscillatory technique on acute hemiplegic shoulder pain". International Journal of Health Sciences and Research 5.7 (2015): 224-233.

38. Kumar P. "The Effects of Lycra Arm Sleeve on Glenohumeral Subluxation in Poststroke Hemiplegia-A Preliminary Study". Journal of Prosthetics and Orthotics 31.1 (2019): 7075 .

39. Lee S and Lim S. "Acupuncture for Poststroke Shoulder Pain: A Systematic Review and Meta-Analysis". (2019).

40. Lee J., et al. "Acupuncture for Shoulder Pain After Stroke: A Systematic Review".The Journal of Alternative and Complementary Medicine 18.9 (2012): 818-823.

41. Pan R., et al. "A randomized controlled trial of a modified wheelchair arm-support to reduce shoulder pain in stroke patients". Clinical Rehabilitation 32.1 (2017): 37-47.
42. Kim M., et al. "Radiographic Imaging Analysis after Sling Exercises for Hemiplegic Shoulder Subluxation". Journal of Physical Therapy Science 24.11 (2012): 1099-1101.

43. Dohle., et al. "Pilot study of a robotic protocol to treat shoulder subluxation in patients with chronic stroke". Journal of NeuroEngineering and Rehabilitation 10 (2013): 88.

44. Kumar P., et al. "Risk factors for inferior shoulder subluxation in patients with stroke". Physical Therapy Reviews 15.1 (2010): 3-11.

45. Wilson RD., et al. "Peripheral nerve stimulation compared with usual care for pain relief of hemiplegic shoulder pain: a randomized controlled trial". American Journal of Physical Medicine and Rehabilitation 93.1 (2014): 17-28.

46. Shin B and Lee M. "Effects of Aromatherapy Acupressure on Hemiplegic Shoulder Pain And Motor Power in Stroke Patients: A Pilot Study". The Journal of Alternative and Complementary Medicine 13.2 (2007): 247-252.

47. Fisher C and Curry H. "Pure Motor Hemiplegia of Vascular Origin”.Archives of Neurology 13 (1965): 30-44.

48. Sullivan s and Schmitz T. "Physical Rehabilitation". 5th ed. Philadelphia: F.A. Davis Company (2007).

49. The World Health Organization MONICA Project (monitoring trendsand determinants in cardiovascular disease): a major internationalcollaboration. WHO MONICA Project Principal Investigators. Journal of Clinical Epidemiology 41 (1988): 105-114.

50. John Chae MD and David Yu MD. "A Critical Review of Neuromuscular Electrical Stimulation for Treatment of Motor Dysfunction in Hemiplegia, Assistive Technology:". The Official Journal of RESNA 12.1 (2000): 33-49.

51. Stolzenberg D., et al. "Current and Future Interventions for Glenohumeral Subluxation in Hemiplegia Secondary to Stroke". Topics in Stroke Rehabilitation 19.5 (2012): 444-456.

52. Turner-Stokes L and Jackson D. "Shoulder pain after stroke: a review of the evidence base to inform the development of an integrated care pathway". Clinical Rehabilitation 16.3 (2002): 276-298.

53. Brooke MM., et al. "Shoulder subluxation in hemipiegia: effects of three different supports". Archives of Physical Medicine and Rehabilitation 72.8 (1991): 582-586.

54. Adey-Wakeling Z., et al. "Hemiplegic Shoulder Pain Reduces Quality of Life After Acute Stroke”. American Journal of Physical Medicine and Rehabilitation 95.10 (2016): 758-763.

\section{Volume 2 Issue 9 September 2019 (C) All rights are reserved by Muhammad Tahir Aslam and Syed Shahzad Ali.}

